\title{
Dispatcher-assisted, compression-only bystander cardiopulmonary resuscitation does not improve the outcomes of unwitnessed out-of-hospital cardiac arrest: An observational study with component analysis of rescue breath combination and dispatcher-assisted instruction
}

Yoshinori Ito ( $\sim$ t99s02y05@gmail.com)

Kanazawa Medical University: Kanazawa Ika Daigaku https://orcid.org/0000-0002-6147-6104

\section{Hideo Inaba}

Kanazawa Ika Daigaku

\section{Tomoyuki Ushimoto}

Kanazawa Ika Daigaku

Hideki Morita

Kanazawa Ika Daigaku

Kenshi Murasaka

Kanazawa Ika Daigaku

\section{Yukihiro Wato}

Kanazawa Ika Daigaku

Original research

Keywords: bystander cardiopulmonary resuscitation, dispatch-assisted CPR, unwitnessed out-of-hospital cardiac arrest, bystander-witnessed out-of-hospital cardiac arrest

Posted Date: April 9th, 2021

DOI: https://doi.org/10.21203/rs.3.rs-388457/v1

License: (c) (i) This work is licensed under a Creative Commons Attribution 4.0 International License. Read Full License 


\section{Abstract}

Objectives: This study aimed to analyse the effects of rescue breath and chest compression combinations in bystander cardiopulmonary resuscitation (BCPR) with and without dispatch-assisted CPR (DA) on the outcomes between unwitnessed and bystander-witnessed out-of-hospital cardiac arrest (OHCA).

Design and Settings: This retrospective study analysed the prospectively collected data of 212,003 unwitnessed and 117,920 bystander-witnessed OHCA cases between 2014 and 2016 in Japan, with BCPR classification based on two clinical components (DA provision [with or without DA] and combination of breaths and compressions [standard or compression-only]).

Main outcome measures: Neurologically favourable outcome at 1 month

Results: In univariate analysis, unwitnessed cases had no significant association of BCPR with the overall neurologically favourable outcome (provided vs not provided, 0.65\% [686/106,152] vs $0.66 \%[694 / 105,851]$ ) compared with bystanderwitnessed cases $(5.6 \%[3,538 / 62,814]$ vs $3.5 \%[1,911 / 55,106])$. After BCPR classification by two clinical components, the outcome of unwitnessed cases was improved by standard BCPR with DA (0.88\% [69/7,807], adjusted OR; $95 \% \mathrm{Cl}, 1.38$; $1.05-1.81)$ and compression-only (1.04\% [161/15,497], 1.49;1.23-1.80) and standard $(1.18 \%[41 / 3,463], 1.71 ; 1.21-$ 2.43) BCPR without DA, but not by compression-only BCPR with DA $(0.52 \%[415 / 79,385], 0.88 ; 0.76-1.01)$. According to multivariable logistic regression analysis focusing on the two clinical components only in cases with $\mathrm{BCPR}$, neurologically favourable outcomes were worse in DA provision $(0.76 ; 0.60-0.97)$ but better in standard $B C P R,(1.27$; 1.01-1.60) without significant interaction $(P=0.16)$, in unwitnessed cases. In bystander-witnessed cases, DA provision was associated with better outcomes $(1.27 ; 1.01-1.60)$, with significant interaction $(P=0.03)$.

Conclusions: Compared with no BCPR, compression-only BCPR with DA does not improve the neurologically favourable outcomes, and standard BCPR without DA is ideal in unwitnessed OHCA cases. Education on standard CPR and chest compression-only CPR as an option should be maintained because numerous OHCA cases are not witnessed by bystanders.

\section{Summary Boxes}

\section{What is already known on this topic:}

Current basic life support guidelines recommend compression-only cardiopulmonary resuscitation (CPR) for untrained rescuers.

Compression-only CPR is likely to be as effective as standard CPR.

Dispatchers are recommended to instruct compression-only CPR for untrained bystanders who need DA instruction.

\section{What this study adds}

Compared with no BCPR, compression-only BCPR with DA does not improve the neurologically favourable outcomes in unwitnessed OHCA cases.

Standard BCPR without DA is adequate in unwitnessed OHCA cases.

Education on standard CPR and compression-only CPR as an option should be maintained.

\section{Introduction}


Current basic life support guidelines recommend compression-only cardiopulmonary resuscitation (CPR) for untrained rescuers $[1,2]$. Although previous observational studies compared the effects of compression-only bystander-initiated CPR (BCPR) with those of the standard (rescue breaths and chest compressions) BCPR on the outcomes of out-ofhospital cardiac arrests (OHCAs) in a large cohort, most of them were conducted on bystander-witnessed cases only [37]. The outcomes of unwitnessed OHCAs are considerably worse than those of bystander-witnessed cases [8], and more OHCA cases are unwitnessed by bystanders, particularly in residential locations [9]. Dispatch-assisted CPR (DA) was more frequently attempted in unwitnessed cases than in witnessed cases [10], presumably because of higher incidences of apparent cardiac arrest signs and lower incidences of agonal breathing interfering with cardiac arrest recognition.

Theoretically, during the first few minutes of OHCA, rescue breaths are less important than chest compressions because blood oxygen levels remain high at this time. Therefore, compression-only CPR may be more effective for a witnessed OHCA, especially in a community with a short emergency medical service (EMS) response time interval, whereas standard CPR is preferred for an unwitnessed OHCA or for an OHCA occurring in a community with a long EMS response time interval $[7,11]$.

The investigation on whether the advantage or disadvantages of compression-only BCPR may differ between cases with and without DA has remained rarely conducted. One previous study [12] classified BCPR into four groups based on BCPR type (compression-only or standard) and initiation (with or without DA) and reported the advantage of standard BCPR without DA in a remote area with a long EMS response time interval.

This study aimed to analyse the effects of the combination of rescue breaths and chest compressions in BCPR and DA on the outcomes of unwitnessed and bystander-witnessed OHCA cases. We compared the outcomes of the four BCPR groups (compression-only with DA, standard with DA, compression-only BCPR without DA and standard BCPR without DA) with those of the no-BCPR group. Then, we conducted a component analysis only in cases with BCPR to reveal the effects of the BCPR type and DA.

\section{Methods}

\section{Study design and setting}

After obtaining consent from the Japanese Fire and Disaster Management Agency (FDMA), we retrospectively analysed their OHCA data prospectively collected between 2014 and 2016 using a nationwide, population-based, all-Japan registry system. The review board of Ishikawa Medical Control Council approved this study. Considering that the database analysed in this study was anonymous and secondary, an informed consent from each patient was waived according to the guidelines in Japan [13].

The Japanese EMS responds to all requests for ambulance dispatch. EMS generally provides DA according to the FDMA protocol [14]. In this protocol, dispatchers are recommended to instruct compression-only CPR to bystanders who are untrained or unwilling to perform rescue breathings. Paramedics in ambulance teams are allowed to use several resuscitation methods, including semi-automated external defibrillation, suprapharyngeal airway device insertion and Ringer's lactate solution infusion via the peripheral vein. For OHCA cases aged $\geq 8$ years, authorised paramedics have been allowed to insert tracheal tubes and administer intravenous epinephrine under online medical direction since 2004 and 2006, respectively. They have also been allowed to perform fluid resuscitation in patients with shock and those with suspected crush syndrome, since 2014. EMS personnel are not allowed to terminate resuscitation until their arrival at hospital.

\section{Data selection}


The FDMA database included the following data based on the Utstein recommendations[15]: Patient age, sex, witness status, OHCA aetiology (presumed cardiac or non-cardiac), initial electrocardiogram rhythm (shockable or nonshockable), public access defibrillation (PAD), any prehospital defibrillation, time of day for emergency call (night time [10:00 PM-5:59 AM] or other), advanced airway management, tracheal intubation, physician in ambulance, advanced life support (ALS) by physician, time interval between emergency call and first CPR performed by the bystander or EMS personnel (whichever was earlier [call-to-first CPR interval or CPR-free time]), time interval between emergency call and EMS contact to patient (EMS response time) and time interval between EMS contact to patient and arrival at hospital BCPR type, DA provision, recorded time of BCPR initiation, emergency call, EMS vehicle arrival, EMS contact to patient, EMS CPR initiation and neurologically favourable (cerebral performance category [CPC] = 1 or 2 ) outcome at 1 month[16].

Out of 372,926 OHCAs recorded in 2014-2016, 4,665 unconfirmed arrest cases, including those with ROSC (return of spontaneous circulation) before EMS contact to patients, were excluded. Then, after excluding 29,987 EMS-witnessed cases, 5,665 cases with incomplete time record and 2,686 cases of child ( $<8$ years) OHCA, we finally included 212,003 unwitnessed cases and 117,920 bystander-witnessed cases (Fig. 1 upper part).

\section{Study endpoints}

The primary endpoint was a neurologically favourable outcome, which was defined as a CPC score of 1 or 2 [16] at 1 month (1-M). The secondary endpoint was 1-M survival.

\section{Classification of prehospital epinephrine administration}

According to the preliminary analysis for the cut-off values of timing (time interval between the EMS initiation of CPR and the first epinephrine administration) and the number of prehospital epinephrine administrations, we classified the epinephrine administrations into the following three groups: no administration, early $(<13 \mathrm{~min}$ after the EMS initiation of CPR) single administration and others.

\section{Definition and classification of BCPR}

BCPR was defined as chest compressions with and without rescue breaths provided by untrained rescuers on OHCA victims before EMS contact. BCPR was classified into the following four groups according to the combination of rescue breaths and chest compressions (compression-only or standard) and DA provision (BCPR with DA or BCPR without DA): compression-only with DA, standard with DA, compression-only BCPR without DA and standard BCPR without DA.

\section{Statistical analysis}

The background and clinical characteristics between groups were compared using the chi-square or Fisher's exact probability tests for nominal variables and the Mann-Whitney $U$ test for continuous variables. The association of BCPR with the outcomes was assessed by multivariate logistic regression analyses including the following factors that were known to be associated with outcomes : the patient characteristics included age, sex, witness status, OHCA aetiology (presumed cardiac or non-cardiac), initial electrocardiogram rhythm (shockable or non-shockable), any prehospital defibrillation, time of day for emergency call (night time [10:00 PM-5:59AM] or other), advanced airway management, epinephrine administration, and time interval between emergency call and EMS contact to patient (EMS response time). Physician in ambulance, advanced life support (ALS) by physicians, time interval between emergency call and first CPR (either by bystanders or EMS), time interval between EMS contact to patients and arrival at hospitals were included in the analysis when one or more of these factors lowered the value of Akaike information Criterion (AIC). All statistical analyses were performed using the JMP® Pro 15 software (SAS Institute, Cary, NC, USA). Using the profile likelihood, we calculated the odds ratios (ORs) and 95\% confidence intervals (Cls). All tests were two tailed, and $P<0.05$ was considered statistically significant. 


\section{Patient and public involvement}

No patient involved in study design and study conduct.

\section{Results}

\section{Overview of DA-CPR and BCPR (Fig. 1, lower part)}

Unwitnessed cases (62.6\%) had more frequent DA attempts than bystander-witnessed cases (52.3\%) (unadjusted OR [95\% Cl]: 1.53 [1.50-1.55]). Regardless of DA provision, bystander-witnessed cases (72.4\%, with DA; $32.0 \%$, without DA) more frequently received BCPR than unwitnessed cases (65.7\%, with DA; $23.9 \%$, without DA) (1.39 [1.36-1.43] and 1.50 [1.46-1.53], respectively). In the groups with BCPR provision, unwitnessed cases (91.1\%, with DA; $81.7 \%$, without DA) more frequently received compression-only BCPR than bystander-witnessed cases $(39.6 \%$, with DA; $77.1 \%$, without DA) regardless of DA attempts (1.37 [1.32-1.42] and 1.33 [1.26-1.40], respectively).

\section{Beneficial effects of BCPR on outcomes}

BCPR improved the overall outcomes, with a significant interaction with witness status (Table 1). The outcomes of bystander-witnessed cases were considerably better than those of unwitnessed cases. Thus, BCPR significantly improved the outcomes mainly in bystander-witnessed cases. 
Table 1

Association of bystander CPR with outcomes of OHCA

\begin{tabular}{|c|c|c|c|c|}
\hline \multirow[t]{2}{*}{$\begin{array}{l}\text { Characteristic and } \\
\text { outcomes }\end{array}$} & \multicolumn{2}{|c|}{$\begin{array}{l}\text { Bystander CPR } \\
\text { (Chest compressions with and } \\
\text { without rescue breathing by } \\
\text { bystanders) }\end{array}$} & \multirow[t]{2}{*}{$\begin{array}{l}\text { Unadjusted OR } \\
\text { (95\% Cl) with no } \\
\text { bystander CPR as } \\
\text { reference }\end{array}$} & \multirow[t]{2}{*}{$\begin{array}{l}\text { Adjusted OR with no } \\
\text { bystander CPR as } \\
\text { reference }^{\text {a) }}\end{array}$} \\
\hline & $\begin{array}{l}\text { Not provided by } \\
\text { bystanders }\end{array}$ & $\begin{array}{l}\text { Provided by } \\
\text { bystanders }\end{array}$ & & \\
\hline \multicolumn{5}{|l|}{$\begin{array}{l}\text { All EMS-unwitnessed } \\
\text { OHCAs }(N=329,923)\end{array}$} \\
\hline $\mathrm{N}$ & 160,957 & 168,966 & & \\
\hline 1-M survival, \% (N) & $3.9(6,179)$ & $4.9(8,214)$ & $1.28(1.24-1.32)$ & $1.11(1.06-1.15)^{b)}$ \\
\hline $\begin{array}{l}\text { Neurologically } \\
\text { favourable outcome, } \\
\%(N)\end{array}$ & $1.6(2,605)$ & $2.5(4,224)$ & $1.56(1.48-1.64)$ & $1.23(1.16-1.31)^{c)}$ \\
\hline \multicolumn{5}{|l|}{$\begin{array}{l}\text { Unwitnessed OHCAs } \\
(N=212,003)\end{array}$} \\
\hline $\mathrm{N}$ & 105,851 & 106,152 & & \\
\hline 1-M survival, \% (N) & $1.8(1,900)$ & $1.8(1,858)$ & $0.97(0.91-1.04)$ & $0.90(0.64-1.28)$ \\
\hline $\begin{array}{l}\text { Neurologically } \\
\text { favourable outcome, } \\
\%(N)\end{array}$ & $0.66(694)$ & $0.65(686)$ & $0.99(0.89-1.10)$ & $1.13(0.99-1.29)$ \\
\hline \multicolumn{5}{|l|}{$\begin{array}{l}\text { Bystander-witnessed } \\
\text { OHCAs }(N=117,920)\end{array}$} \\
\hline $\mathrm{N}$ & 55,106 & 62,814 & & \\
\hline 1-M survival, \% (N) & $7.8(4,279)$ & $10.1(6,356)$ & $1.34(1.28-1.39)$ & $1.29(1.21-1.38)$ \\
\hline $\begin{array}{l}\text { Neurologically } \\
\text { favourable outcome, } \\
\%(N)\end{array}$ & $3.5(1,911)$ & $5.6(3,538)$ & $1.66(1.57-1.76)$ & $1.46(1.36-1.57)$ \\
\hline
\end{tabular}

OHCA: out-of-hospital cardiac arrest, CPR: cardiopulmonary resuscitation, OR: odds ratio, Cl: confidence interval, DACPR: dispatcher-assisted CPR instruction.

a) Multiple variable regression analyses included the followings as candidate for the final model with the least Akaike Information Criterion: patient age, sex, emergency call during nighttime (10:00 p.m.-6:00 a.m.), bystander CPR (chest compressions), shockable initial rhythm, public access defibrillation, any prehospital defibrillation, presumed cardiac etiology, advanced airway management, tracheal intubation, classification of epinephrine administration (no epinephrine, early [EMS initiation of CPR-first administration < $13 \mathrm{~min}$ ] single-dose administration and other administrations), and time intervals of emergency call-to-first CPR interval, call-to- EMS contact to patient and and EMS contact to patient-to-EMS arrival at hospital interval. In the analysis for all EMS-unwitnessed OHCAs, witness and its interaction with bystander CPR were also included.

b) Significant interaction between witness and bystander CPR $(P<0.01)$. adjusted $O R ; 95 \% \mathrm{Cl}$ with unwitnessed cases as reference: $3.02 ; 2.85-3.19$.

c) Significant interaction between witness and bystander CPR $(\mathrm{P}<0.01)$. adjusted OR; $95 \% \mathrm{Cl}$ with unwitnessed cases as reference: $2.76 ; 2.28-3.26$.

Comparisons of the outcomes of the four BCPR groups with those without BCPR in unwitnessed and bystanderwitnessed cases 
In unwitnessed cases, lower rates of neurologically favourable outcomes $(0.52 \%$ vs $0.66 \%$, adjusted OR [ $95 \% \mathrm{Cl}$ ], 0.88 [0.76-1.01]) and lower 1-M survival rate (1.5\% vs $1.8 \%, 0.89$ [0.81-0.97]) were observed in compression-only BCPR with DA compared with those in no BCPR (Fig. 2A). The rates of neurologically favourable outcome and 1-M survival were highest in standard BCPR without DA (1.2\% and $2.5 \%$, respectively).

In bystander-witnessed cases, higher rates of neurologically favourable outcomes and 1-M survival were observed in all four BCPR groups compared with those without BCPR (Fig. 2B). The rates of neurologically favourable outcomes and 1M survival were highest in standard BCPR with DA (6.6\% and $10.9 \%$, respectively).

Component analyses of the combination of rescue breaths and chest compressions and DA provision in OHCA cases receiving $B C P R$

When the characteristics of unwitnessed OHCA cases receiving BCPR were compared between groups with and without DA-CPR (Table 2, left part), the differences were remarkable (unadjusted OR of $<0.8$ or $>1.25$ for nominal variables and median value for continuous variables) in terms of age, PAD, any prehospital defibrillation, advanced airway management, ALS by physician and time interval between emergency call and first CPR. Cases with DA were younger and less frequently had PAD, prehospital defibrillation, advanced airway management and ALS by physician than those without DA. However, BCPR initiation was delayed in cases with DA. 
Association between clinical characteristics of BCPR and OHCA in unwitnessed cases with bystander CPR

\begin{tabular}{|c|c|c|c|c|c|c|}
\hline \multirow{3}{*}{$\begin{array}{l}\text { Clinical } \\
\text { characteristics and } \\
\text { outcomes }\end{array}$} & \multicolumn{6}{|c|}{ Characteristics of bystander CPR } \\
\hline & \multicolumn{3}{|l|}{ DA-CPR } & \multicolumn{3}{|c|}{$\begin{array}{l}\text { Combination of ventilations with chest } \\
\text { compressions in BCPR }\end{array}$} \\
\hline & $\begin{array}{l}\text { BCPR } \\
\text { with DA- } \\
\text { CPR }\end{array}$ & $\begin{array}{l}\text { BCPR } \\
\text { without } \\
\text { DA-CPR }\end{array}$ & $\begin{array}{l}\text { Unadjusted OR } \\
(95 \% \mathrm{Cl}) \text { with } \\
\mathrm{BCPR} \text { without } \mathrm{DA}- \\
\mathrm{CPR} \text { as reference } \\
\text { or } \mathrm{P} \text { value }\end{array}$ & Standard & $\begin{array}{l}\text { Compression- } \\
\text { only }\end{array}$ & $\begin{array}{l}\text { Unadjusted } \\
\text { OR }(95 \% \mathrm{Cl}) \\
\text { with } \\
\text { compression- } \\
\text { only } \mathrm{BCPR} \text { as } \\
\text { reference } \\
\text { or } \mathrm{P} \text { value }\end{array}$ \\
\hline $\mathrm{N}$ & 87,192 & 18,960 & & 11,270 & 94,882 & \\
\hline Male, \% (N) & $\begin{array}{l}51.6 \% \\
(44,978)\end{array}$ & $\begin{array}{l}50.8 \% \\
(9,627)\end{array}$ & $1.03(1.01-1.07)$ & $\begin{array}{l}45.5 \% \\
(5,131)\end{array}$ & $\begin{array}{l}52.1 \% \\
(49,474)\end{array}$ & $\begin{array}{l}0.77(0.74- \\
0.80)\end{array}$ \\
\hline Age, y, median (IQR) & $\begin{array}{l}81(70- \\
87)\end{array}$ & $\begin{array}{l}82(70- \\
88)\end{array}$ & $<0.01$ & $\begin{array}{l}82(70- \\
88)\end{array}$ & $81(70-87)$ & $<0.01$ \\
\hline $\begin{array}{l}\text { Emergency call } \\
\text { during nighttime } \\
(10: 00 \text { p.m. }-5: 59 \\
\text { a.m.), \% (N) }\end{array}$ & $\begin{array}{l}21.9 \% \\
(19,058)\end{array}$ & $\begin{array}{l}22.0 \% \\
(4,162)\end{array}$ & $0.99(0.96-1.03)$ & $\begin{array}{l}21.4 \% \\
(2,408)\end{array}$ & $\begin{array}{l}21.9 \% \\
(20,812)\end{array}$ & $\begin{array}{l}0.97(0.93- \\
1.01)\end{array}$ \\
\hline $\begin{array}{l}\text { Of presumed } \\
\text { cardiac etiology, \% } \\
\text { (N) }\end{array}$ & $\begin{array}{l}64.2 \% \\
(55,978)\end{array}$ & $\begin{array}{l}62.6 \% \\
(11,872)\end{array}$ & $1.07(1.04-1.11)$ & $\begin{array}{l}63.1 \% \\
(7,113)\end{array}$ & $\begin{array}{l}64.0 \% \\
(60,706)\end{array}$ & $\begin{array}{l}0.96(0.92- \\
1.08)\end{array}$ \\
\hline $\begin{array}{l}\text { Shockable initial } \\
\text { rhythm, \% (N) }\end{array}$ & $\begin{array}{l}3.1 \% \\
(2,693)\end{array}$ & $\begin{array}{l}3.8 \% \\
(718)\end{array}$ & $0.81(0.74-0.88)$ & $\begin{array}{l}3.7 \% \\
(411)\end{array}$ & $\begin{array}{l}3.2 \% \\
(3000)\end{array}$ & $\begin{array}{l}1.16(1.04- \\
1.29)\end{array}$ \\
\hline $\begin{array}{l}\text { Any prehospital } \\
\text { defibrillation, \% (N) }\end{array}$ & $\begin{array}{l}6.1 \% \\
(5,324)\end{array}$ & $\begin{array}{l}8.2 \% \\
(1,545)\end{array}$ & $0.73(0.69-0.78)$ & $\begin{array}{l}9.0 \% \\
(1,018)\end{array}$ & $\begin{array}{l}6.2 \% \\
(5,891)\end{array}$ & $\begin{array}{l}1.51(1.41- \\
1.62)\end{array}$ \\
\hline $\begin{array}{l}\text { Public access } \\
\text { defibrillation,\% (N) }\end{array}$ & $\begin{array}{l}0.9 \% \\
(818)\end{array}$ & $\begin{array}{l}2.9 \% \\
(557)\end{array}$ & $0.31(0.28-0.35)$ & $\begin{array}{l}3.7 \% \\
(412)\end{array}$ & $\begin{array}{l}1.0 \% \\
(963)\end{array}$ & $\begin{array}{l}3.70(3.29- \\
4.16)\end{array}$ \\
\hline $\begin{array}{l}\text { Advanced airway } \\
\text { management, \% }(\mathrm{N})\end{array}$ & $\begin{array}{l}42.7 \% \\
(87,192)\end{array}$ & $\begin{array}{l}35.9 \% \\
(18,960)\end{array}$ & $1.33(1.29-1.38)$ & $\begin{array}{l}40.9 \% \\
(4,604)\end{array}$ & $\begin{array}{l}41.6 \% \\
(39,470)\end{array}$ & $\begin{array}{l}0.97(0.93- \\
1.01)\end{array}$ \\
\hline $\begin{array}{l}\text { Tracheal intubation, } \\
\%(\mathrm{~N})\end{array}$ & $\begin{array}{l}7.7 \% \\
(6,673)\end{array}$ & $\begin{array}{l}7.5 \% \\
(1,423)\end{array}$ & $1.02(0.96-1.08)$ & $\begin{array}{l}8.1 \% \\
(912)\end{array}$ & $\begin{array}{l}7.6 \% \\
(7,184)\end{array}$ & $\begin{array}{l}1.07(1.00- \\
1.15)\end{array}$ \\
\hline $\begin{array}{l}\text { Prehospital epinephri } \\
\text { (N) }\end{array}$ & administr & tion, \% & & & & \\
\hline No & $\begin{array}{l}85.9 \% \\
(74,912)\end{array}$ & $\begin{array}{l}86.8 \% \\
(16,463)\end{array}$ & $<0.01$ & $\begin{array}{l}84.4 \% \\
(9,512)\end{array}$ & $\begin{array}{l}86.3 \% \\
(81,863)\end{array}$ & $<0.01$ \\
\hline
\end{tabular}

OHCA: out-of-hospital cardiac arrest, CPR: cardiopulmonary resuscitation, OR: odds ratio, Cl: confidence interval, BCPR: bystander CPR, DA-CPR: dispatcher-assisted CPR instruction, PAD: Public access defibrillation, EMS: emergency medical service, ALS: advanced life support. 


\begin{tabular}{|c|c|c|c|c|c|c|}
\hline \multirow{3}{*}{$\begin{array}{l}\text { Clinical } \\
\text { characteristics and } \\
\text { outcomes }\end{array}$} & \multicolumn{6}{|c|}{ Characteristics of bystander CPR } \\
\hline & \multicolumn{3}{|l|}{ DA-CPR } & \multicolumn{3}{|c|}{$\begin{array}{l}\text { Combination of ventilations with chest } \\
\text { compressions in BCPR }\end{array}$} \\
\hline & $\begin{array}{l}\text { BCPR } \\
\text { with DA- } \\
\text { CPR }\end{array}$ & $\begin{array}{l}\text { BCPR } \\
\text { without } \\
\text { DA-CPR }\end{array}$ & $\begin{array}{l}\text { Unadjusted OR } \\
(95 \% \mathrm{Cl}) \text { with } \\
\mathrm{BCPR} \text { without } \mathrm{DA}- \\
\mathrm{CPR} \text { as reference } \\
\text { or } \mathrm{P} \text { value }\end{array}$ & Standard & $\begin{array}{l}\text { Compression- } \\
\text { only }\end{array}$ & $\begin{array}{l}\text { Unadjusted } \\
\text { OR }(95 \% \mathrm{CI}) \\
\text { with } \\
\text { compression- } \\
\text { only BCPR as } \\
\text { reference } \\
\text { or } \mathrm{P} \text { value }\end{array}$ \\
\hline \multirow{2}{*}{$\begin{array}{l}\text { Early single } \\
\text { administration }\end{array}$} & $2.3 \%$ & $2.2 \%$ & & $2.9 \%$ & $2.2 \%$ & \\
\hline & $(2,020)$ & $(422)$ & & $(328)$ & $(2,114)$ & \\
\hline \multirow{2}{*}{$\begin{array}{l}\text { Other } \\
\text { administrations }\end{array}$} & $11.8 \%$ & $10.9 \%$ & & $12.7 \%$ & $11.5 \%$ & \\
\hline & $(2,075)$ & $(2,075)$ & & $(1,430)$ & $(10,905)$ & \\
\hline \multirow{2}{*}{$\begin{array}{l}\text { Physician in } \\
\text { ambulance, \% (N) }\end{array}$} & $2.4 \%$ & $2.9 \%$ & \multirow[t]{2}{*}{$0.81(0.74-0.89)$} & $3.6 \%$ & $2.4 \%$ & \multirow{2}{*}{$\begin{array}{l}1.53(1.37- \\
1.70)\end{array}$} \\
\hline & $(2,082)$ & $(555)$ & & $(401)$ & $(2,236)$ & \\
\hline \multirow{2}{*}{$\begin{array}{l}\text { ALS by physician, } \% \\
\text { (N) }\end{array}$} & $8.0 \%$ & $10.6 \%$ & \multirow[t]{2}{*}{$0.74(0.70-0.78)$} & $10.1 \%$ & $8.3 \%$ & \multirow{2}{*}{$\begin{array}{l}1.25(1.17- \\
1.33)\end{array}$} \\
\hline & $(6,970)$ & $(2,000)$ & & $(1,138)$ & $(7,832)$ & \\
\hline \multicolumn{7}{|c|}{ Time intervals, min, median (IQR) } \\
\hline $\begin{array}{l}\text { Emergency call-to- } \\
\text { BCPR (first CPR) }\end{array}$ & $1(0-2)$ & $0(-2-2)$ & $<0.01$ & $0(-3-1)$ & $1(0-2)$ & $<0.01$ \\
\hline $\begin{array}{l}\text { EMS response time } \\
\text { interval }\end{array}$ & $\begin{array}{l}9(7- \\
11)\end{array}$ & $\begin{array}{l}9(7- \\
11)\end{array}$ & 0.85 & $9(7-11)$ & $9(7-11)$ & 0.54 \\
\hline $\begin{array}{l}\text { EMS contact to } \\
\text { patient-to-arrival at } \\
\text { hospitals }\end{array}$ & $\begin{array}{l}22(17- \\
28)\end{array}$ & $\begin{array}{l}22(17- \\
29)\end{array}$ & $<0.01$ & $\begin{array}{l}22(17- \\
28)\end{array}$ & $22(17-28)$ & 0.62 \\
\hline
\end{tabular}

When the characteristics of unwitnessed OHCA cases receiving BCPR were compared between compression-only and standard BCPR groups (Table 2, right part), cases with compression-only BCPR were younger and less frequently had PAD, prehospital defibrillation and ALS in ambulance than those with standard BCPR. However, BCPR initiation was delayed in cases with compression-only BCPR.

Some characteristics of bystander-witnessed cases differed between the BCPR groups. Notably, the incidence of shockable rhythm in bystander-witnessed cases was higher in the groups receiving BCPR with DA and compression-only $B C P R$, whereas that in unwitnessed cases was slightly higher in the groups receiving BCPR without DA and standard BCPR (Supplementary Table 1).

According to simple binominal logit analyses with interaction test, BCPR with DA was associated with less neurologically favourable outcome and worse 1-M survival in unwitnessed cases; meanwhile, standard BCPR was associated with better outcomes in both unwitnessed and bystander-witnessed cases receiving BCPR (Table 3). A significant interaction was found between DA provision and standard BCPR for 1-M survival in unwitnessed cases $(P=0.01)$ and for neurologically favourable outcomes in bystander-witnessed cases $(P=0.01)$. 
Table 3

Associations of clinical characteristics of bystander CPR with outcomes of OHCA (Simple binominal logit analysis with interaction test)

\begin{tabular}{|c|c|c|c|c|c|c|c|}
\hline \multirow{3}{*}{$\begin{array}{l}\text { Outcomes of } \\
\text { OHCA }\end{array}$} & \multicolumn{6}{|c|}{ Characteristics of bystander CPR } & \multirow{3}{*}{$\begin{array}{l}\text { Interaction of } \\
\text { the two } \\
\text { bystander } \\
\text { CPR } \\
\text { characteristics }\end{array}$} \\
\hline & \multicolumn{3}{|l|}{ DA-CPR } & \multicolumn{3}{|c|}{$\begin{array}{l}\text { Combination of rescue } \\
\text { breaths with chest } \\
\text { compressions in BCPR }\end{array}$} & \\
\hline & $\begin{array}{l}\text { BCPR } \\
\text { with } \\
\text { DA- } \\
\text { CPR }\end{array}$ & $\begin{array}{l}\text { BCPR } \\
\text { without } \\
\text { DA- } \\
\text { CPR }\end{array}$ & $\begin{array}{l}\text { Adjusted } \\
\text { OR (95\% } \\
\text { Cl) with } \\
\text { BCPR } \\
\text { without } \\
\text { DA-CPR } \\
\text { as } \\
\text { reference }\end{array}$ & Standard & $\begin{array}{l}\text { Compression- } \\
\text { only }\end{array}$ & $\begin{array}{l}\text { Adjusted OR } \\
\text { ( } 95 \% \mathrm{Cl} \text { ) with } \\
\text { Compression- } \\
\text { only BCPR as } \\
\text { reference }\end{array}$ & \\
\hline \multicolumn{8}{|l|}{$\begin{array}{l}\text { Unwitnessed } \\
\text { OHCA }\end{array}$} \\
\hline $\mathrm{N}$ & 18,960 & 87,192 & & 11,270 & 94,882 & & \\
\hline 1-M survival & $\begin{array}{l}1.6 \% \\
(1,406)\end{array}$ & $\begin{array}{l}2.4 \% \\
(452)\end{array}$ & $\begin{array}{l}0.78 \\
(0.68- \\
0.90)\end{array}$ & $\begin{array}{l}2.4 \% \\
(274)\end{array}$ & $1.7 \%(1,584)$ & $\begin{array}{l}1.30(1.13- \\
1.50)\end{array}$ & $P=0.01$ \\
\hline $\begin{array}{l}\text { Neurologically } \\
\text { favorable } \\
\text { outcome }\end{array}$ & $\begin{array}{l}0.56 \% \\
(484)\end{array}$ & $\begin{array}{l}1.1 \% \\
(202)\end{array}$ & $\begin{array}{l}0.61 \\
(0.49- \\
0.76)\end{array}$ & $\begin{array}{l}0.98 \% \\
(110)\end{array}$ & $0.61 \%(576)$ & $\begin{array}{l}1.39(1.12- \\
1.72)\end{array}$ & $P=0.07$ \\
\hline \multicolumn{8}{|l|}{$\begin{array}{l}\text { Witnessed } \\
\text { OHCA }\end{array}$} \\
\hline $\mathrm{N}$ & 44,832 & 17,982 & & 9,422 & 53,392 & & \\
\hline $\begin{array}{l}\text { 1-M survival, } \\
\%(N)\end{array}$ & $\begin{array}{l}10.1 \% \\
(4,525)\end{array}$ & $\begin{array}{l}10.2 \% \\
(1,831)\end{array}$ & $\begin{array}{l}1.01 \\
(0.94- \\
1.09)\end{array}$ & $\begin{array}{l}10.8 \% \\
(1,017)\end{array}$ & $10.0 \%(5,339)$ & $\begin{array}{l}1.08(1.01- \\
1.17)\end{array}$ & $P=0.61$ \\
\hline $\begin{array}{l}\text { Neurologically } \\
\text { favorable } \\
\text { outcome, \% } \\
\text { (N) }\end{array}$ & $\begin{array}{l}5.5 \% \\
(2,467)\end{array}$ & $\begin{array}{l}6.0 \% \\
(1,071)\end{array}$ & $\begin{array}{l}1.00 \\
(0.91- \\
1.10)\end{array}$ & $\begin{array}{l}6.3 \% \\
(595)\end{array}$ & $5.5 \%(2,943)$ & $\begin{array}{l}1.11(1.01- \\
1.22)\end{array}$ & $P=0.01$ \\
\hline $\begin{array}{l}\text { OHCA: out-of-h } \\
\text { BCPR: bystand } \\
\text { analysis includ }\end{array}$ & $\begin{array}{l}\text { oital ca } \\
\text { CPR, D }\end{array}$ & $\begin{array}{l}\text { carrest } \\
\text { R: disp }\end{array}$ & PR: cardio & $\begin{array}{l}\text { lary resus } \\
\text { instructio }\end{array}$ & $\begin{array}{l}\text { ation, OR: odd } \\
\text { Adjusted OR V }\end{array}$ & $\begin{array}{l}\text { tio, Cl: confide } \\
\text { calculated by }\end{array}$ & $\begin{array}{l}\text { e interval, } \\
\text { lominal }\end{array}$ \\
\hline
\end{tabular}

Multivariable analysis on neurologically favourable outcomes (Fig. 3) revealed that BCPR with DA was associated with worse outcomes in unwitnessed cases (adjusted OR [95\% Cl], 0.76 [0.60-0.97]) and better outcomes in bystanderwitnessed cases (1.28 [1.14-1.43]). This Fig. is particularly helpful in getting an at-a-glance summary. However, standard BCPR was associated with better outcomes only in unwitnessed cases (1.27 [1.01-1.60]). Regarding 1-M survival, standard BCPR was associated with higher survival rates in both unwitnessed cases (1.06 [1.02-1.38]) and bystanderwitnessed cases (1.16 [1.07-1.26]). However, DA was associated with higher survival rates (1.16 [1.07-1.26]) only in bystander-witnessed cases (Supplementary Fig. 1). Other major common factors associated with neurologically favourable outcomes were age, initial shockable rhythm, any prehospital defibrillation and response time interval. Notably, early single administration of epinephrine was associated with a higher 1-M survival rate but not with better neurologically favourable outcomes. 


\section{Discussion}

In this study, we compared the outcomes of OHCA cases receiving BCPR, which was categorised into four groups, with the outcomes of those who did not receive BCPR. Compression-only BCPR with DA was associated with better neurologically favourable outcomes than no BCPR in bystander-witnessed cases but not in unwitnessed cases. Furthermore, the component analyses in unwitnessed cases revealed the significant associations of rescue breaths with better neurologically favourable outcomes and of DA provision with worse outcomes, without a significant interaction between the two components. The analyses in bystander-witnessed cases disclosed a significant interaction between the two components and confirmed that DA provision is associated with better neurologically favourable outcomes.

BCPR intervention in unwitnessed OHCA cases with extremely poor outcomes may slightly influence the overall outcomes of OHCA [8]. However, as reported previously [10], DA was more frequently attempted in unwitnessed cases, and more OHCA cases were unwitnessed. Numerous unwitnessed cases are found in residential locations by family members who are mostly untrained for CPR $[11,17]$. Therefore, knowing the effectiveness of compression-only DA in unwitnessed cases is important.

Effectiveness in terms of the outcomes and BCPR performance was compared between compression-only and standard CPR in both previous observational [3-6] and randomised control studies [18]. Although the results varied among observational studies, the differences in outcome were not remarkable, and compression-only BCPR commonly improved the OHCA outcomes compared with no BCPR [2]. However, these analyses were performed in bystander-witness cases or subgroup of presumed cardiac aetiology, not focusing on unwitnessed cases. Furthermore, these previous studies did not evaluate the interaction between rescue breaths and DA provision. In a randomised control study conducted in Sweden, no significant difference was found in 30-day survival, but this study was conducted only in cases with DA provision [18].

The significant associations of rescue breaths with better neurologically favourable outcomes and of DA provision with worse outcomes suggest that standard BCPR without DA was ideal in unwitnessed cases who had terminal depletion of blood and tissue oxygen levels, as reported previously in bystander-witnessed OHCA cases with long EMS response time interval [12] and delayed BCPR [6]. The association of DA with better outcomes and lower incidence of DA provision in bystander-witnessed cases support the adherence to current guideline recommendations [1, 2] in which dispatchers should make every effort to detect OHCA through communication with witnesses or callers and instruct compression-only CPR to untrained bystanders.

The present study has several strengths. Firstly, we focused on the effectiveness of BCPR in unwitnessed cases according to the considerable interaction between witness status and BCPR provision. Secondly, we classified BCPR into four groups by the combination of rescue breaths and DA provision; these groups were subjected to component analyses with interaction test. Thirdly, our data are based on a nationwide registry. However, this study also has several limitations. Firstly, the study covered only the period during which dispatchers were recommended to instruct compression-only CPR to bystanders who were untrained or unwilling to perform rescue breaths. Secondly, bystanders' backgrounds such as age, bystander-patient relationship and training experience and locations of OHCA were not included in the analysis because of lack of these data in unwitnessed cases. Therefore, these factors, which are associated with BCPR quality [17, 19], might affect the study results. Thirdly, the time of collapse or intervals from this time point was unavailable in unwitnessed cases and was not included in the multiple regression analyses. Lastly, a risk of misclassifications for DA and combination of rescue breaths and chest compressions might have occurred.

Increasing the proportion of BCPR without DA in unwitnessed cases may be difficult, considering that an unwitnessed OHCA is mostly discovered by untrained bystanders, such as family members. Recent pandemic with COVID-19 might augment the reluctance to perform rescue breathings [20]. One strategy to resolve this issue is EMS, as well as community interventions, such as recruiting trained volunteers to initiate standard BCPR [21]. Teaching standard CPR 
with high-quality rescue breathings and chest compressions to staff in public facilities, such as care facilities, schools and universities, is also recommended. Education on standard CPR and chest compression-only CPR as an option should be maintained to improve the outcomes of unwitnessed OHCA.

\section{Conclusions}

Compared with no BCPR, compression-only BCPR with DA does not improve the neurologically favourable outcomes, and standard BCPR without DA is adequate in unwitnessed OHCA cases. Education on standard CPR and chest compressiononly CPR as an option should be maintained because numerous OHCA cases are not witnessed by bystanders.

\section{Declarations}

Ethics approval and consent to participate

This study was approved by the institutional review board of the Ishikawa Medical Control Council and conducted by the study group comprising members of the Ishikawa Medical Control Council and their collaborators. Patient consent was not required for use of secondary data. Consent for publication.

Availability of data and material

Data sharing not applicable to this article as no datasets were generated or analysed during the current study.

\section{Competing interests}

The authors declare that they have no competing interests.

Funding

Not applicable.

\section{Authors' contributions}

Contributions: $\mathrm{YI}$ and $\mathrm{HI}$ had full access to all the data in the study and take responsibility for the integrity of the data and the accuracy of the data analysis.

Study concept and design: $\mathrm{YI}$ and $\mathrm{HI}$.

Acquisition of data: all authors.

Analysis and interpretation of data: all authors.

Drafting of the manuscript: $\mathrm{YI}, \mathrm{HI}$ and TU.

Clinical revision of the manuscript for the important intellectual content: YI. HI and YW

Statistical analysis: $\mathrm{YI}$ and $\mathrm{HI}$.

Administrative, technical, or material support: YI. HI and YW.

Study supervision: $\mathrm{HI}$ and $\mathrm{YW}$.

Acknowledgements

Page 12/17 
We would like to thank the EMS personnel and the FDMA in Japan for their great cooperation in collecting and managing the Utstein-style database.

\section{References}

1. Kleinman ME, Goldberger ZD, Rea T, Swor RA, Bobrow BJ, Brennan EE, Terry M, Hemphill R, Gazmuri RJ, Hazinski MF, Travers AH. 2017 American Heart Association Focused Update on Adult Basic Life Support and Cardiopulmonary Resuscitation Quality: An Update to the American Heart Association Guidelines for Cardiopulmonary Resuscitation and Emergency Cardiovascular Care. Circulation 2018;137:e7-e13.

2. Olasveengen TM, de Caen AR, Mancini ME, Maconochie IK, Aickin R, Atkins DL, Berg RA, Bingham RM, Brooks SC, Castrén M, Chung SP, Considine J, Couto TB, Escalante R, Gazmuri RJ, Guerguerian AM, Hatanaka T, Koster RW, Kudenchuk PJ, Lang E, Lim SH, Løfgren B. 2017 International Consensus on Cardiopulmonary Resuscitation and Emergency Cardiovascular Care Science With Treatment Recommendations Summary. Resuscitation 2017;121:201214.

3. Riva G, Ringh M, Jonsson M, Svensson L, Herlitz J, Claesson A, Djärv T, Nordberg P, Forsberg S, Rubertsson S, Nord A, Rosenqvist M, Hollenberg J. Survival in Out-of-Hospital Cardiac Arrest After Standard Cardiopulmonary Resuscitation or Chest Compressions Only Before Arrival of Emergency Medical Services: Nationwide Study During Three Guideline Periods. Circulation 2019;139;2600-2609.

4. Kitamura T, Kiyohara K, Nishiyama C, Kiguchi T, Kobayashi D, Kawamura T, Iwami T. Chest compression-only versus conventional cardiopulmonary resuscitation for bystander-witnessed out-of-hospital cardiac arrest of medical origin: A propensity score-matched cohort from 143,500 patients. Resuscitation 2018;126:29-35.

5. SOS-KANTO study group. Cardiopulmonary resuscitation by bystanders with chest compression only (SOS-KANTO): an observational study. Lancet 2007;369(9565):920-6.

6. Ogawa T, Akahane M, Koike S, Tanabe S, Mizoguchi T, Imamura T. Outcomes of chest compression only CPR versus conventional CPR conducted by lay people in patients with out of hospital cardiopulmonary arrest witnessed by bystanders: nationwide population based observational study. BMJ 2011;342:c7106.

7. Kitamura T, Iwami T, Kawamura T, Nagao K, Tanaka H, Berg RA, Hiraide A; Implementation Working Group for AllJapan Utstein Registry of the Fire and Disaster Management Agency. Time-dependent effectiveness of chest compression-only and conventional cardiopulmonary resuscitation for out-of-hospital cardiac arrest of cardiac origin. Resuscitation 2011;82:3-9.

8. Kuisma M1, Jaara K., Unwitnessed out-of-hospital cardiac arrest: is resuscitation worthwhile? Ann Emerg Med 1997;30:69-75.

9. Okabayashi S, Matsuyama T, Kitamura T, Kiyohara K, Kiguchi T, Nishiyama C, Kobayashi D, Shimamoto T, Sado J, Kawamura T, Iwami T. Outcomes of Patients 65 Years or Older After Out-of-Hospital Cardiac Arrest Based on Location of Cardiac Arrest in Japan. JAMA Netw Open 2019;2:e191011.

10. Tanaka Y, Taniguchi J, Wato Y, Yoshida Y, Inaba H. The continuous quality improvement project for telephoneassisted instruction of cardiopulmonary resuscitation increased the incidence of bystander CPR and improved the outcomes of out-of-hospital cardiac arrests. Resuscitation 2012;83:1235-41.

11. Mickey S. Eisenberg, M.D., "Resuscitate!: How Your Community Can Improve Survival from Sudden Cardiac Arrest" University of Washington Press; Second edition, January 17, 2013.

12. Takei Y, Kamikura T, Nishi T, Maeda T, Sakagami S, Kubo M, Inaba H. Recruitments of trained citizen volunteering for conventional cardiopulmonary resuscitation are necessary to improve the outcome after out-of-hospital cardiac arrests in remote time-distance area: A nationwide population-based study. Resuscitation 2016;105:100-8. 
13. Ministry of Health, Labor and Welfare (in japan). https://www.mhlw.go.jp/general/seido/kousei/ikenkyu/rinsyo/dl/shishin.pdf (in japanese) [accessed 20.04.10]

14. Fire and Disaster Management Agency (in Japan). https://www.fdma.go.jp/laws/laws/laws017.html (in Japanese) [accessed 20.04.10].

15. Jacobs I, Nadkarni V, Bahr J, Berg RA, Billi JE, Bossaert L, Cassan P, Coovadia A, D'Este K, Finn J, Halperin H, Handley A, Herlitz J, Hickey R, Idris A, Kloeck W, Larkin GL, Mancini ME, Mason P, Mears G, Monsieurs K, Montgomery W, Morley P, Nichol G, Nol, Cardiac arrest and cardiopulmonary resuscitation outcome reports: update and simplification of the Utstein templates for resuscitation registries: a statement for healthcare professionals from a task force of the International Liaison Committee on Resuscitation. Circulation 2004;110(21):3385-97.

16. Safar P. "Resuscitation after brain ischemia" Brain failure and resuscitation., 1981. p. 155-84.17.

17. Nishi T, Maeda T, Takase K, Kamikura T, Tanaka Y, Inaba H. Does the number of rescuers affect the survival rate from out-of-hospital cardiac arrests? Two or more rescuers are not always better than one. Resuscitation 2013;84:154-61.

18. Svensson L, Bohm K, Castrèn M, Pettersson H, Engerström L, Herlitz J, Rosenqvist M., Compression-only CPR or standard CPR in out-of-hospital cardiac arrest. N Engl J Med 2010;363(5):434-42.

19. Takei Y, Nishi T, Matsubara H, Hashimoto M, Inaba H., Factors associated with quality of bystander CPR: the presence of multiple rescuers and bystander-initiated CPR without instruction. Resuscitation. 2014;85:492-8.

20. ILCOR COVID-19 Practical Guidance for implementation. https://www.ilcor.org/covid-19 [accessed 20.04.30]

21. Ringh $M$, Rosenqvist M, Hollenberg J, Jonsson M, Fredman D, Nordberg P, Järnbert-Pettersson H, Hasselqvist-Ax I, Riva G, Svensson L. Mobile-phone dispatch of laypersons for CPR in out-of-hospital cardiac arrest. N Engl J Med 2015;372:2316-25.

\section{Figures}




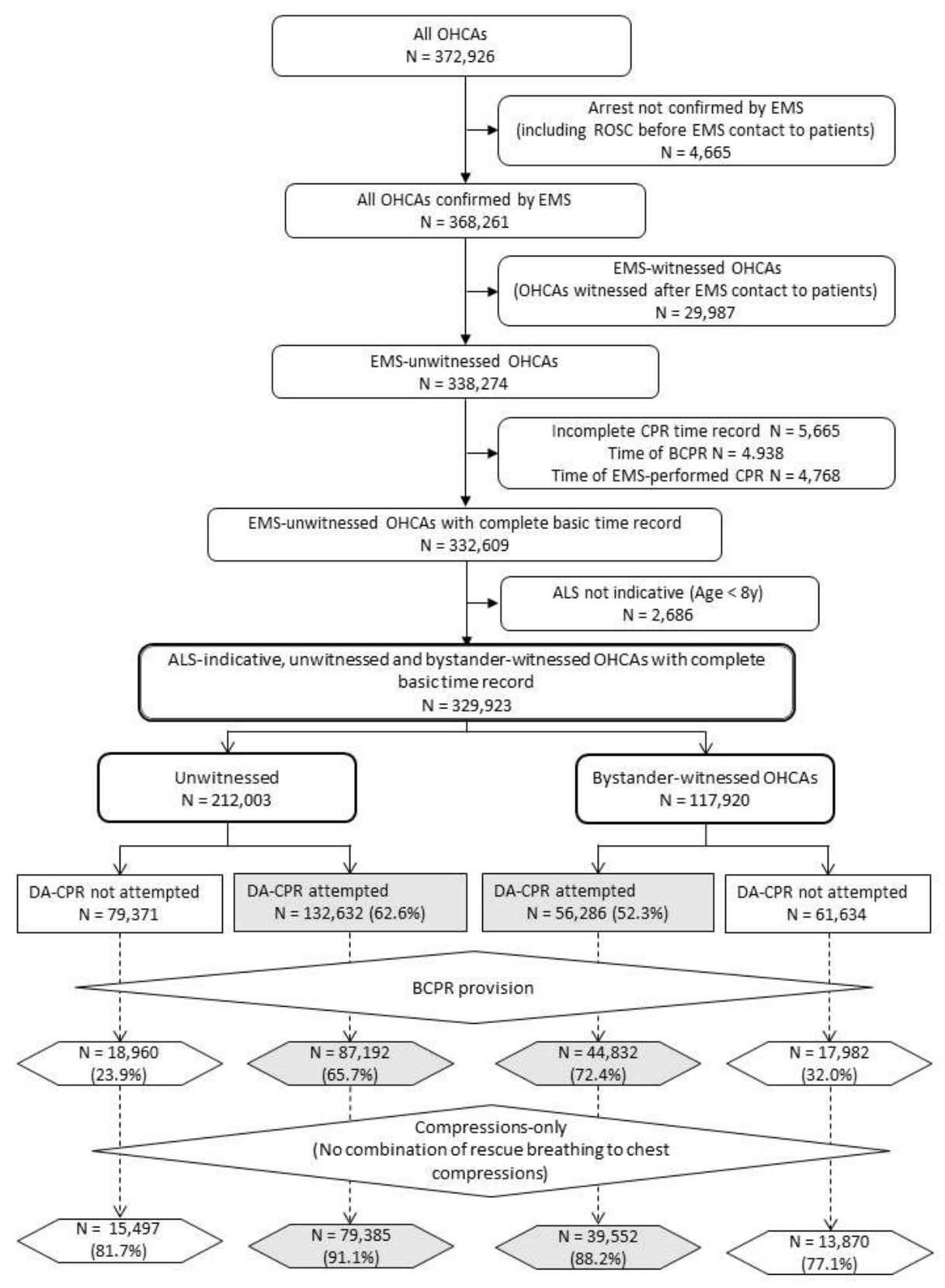

Figure 1

Data selection OHCA: out-of-hospital cardiac arrest, EMS: emergency medical service, ALS: advanced life support, BCPR: bystander cardiopulmonary resuscitation, DA-CPR: dispatcher-assisted CPR. 


\section{A. Unwitnessed OHCAs}

1-M survival

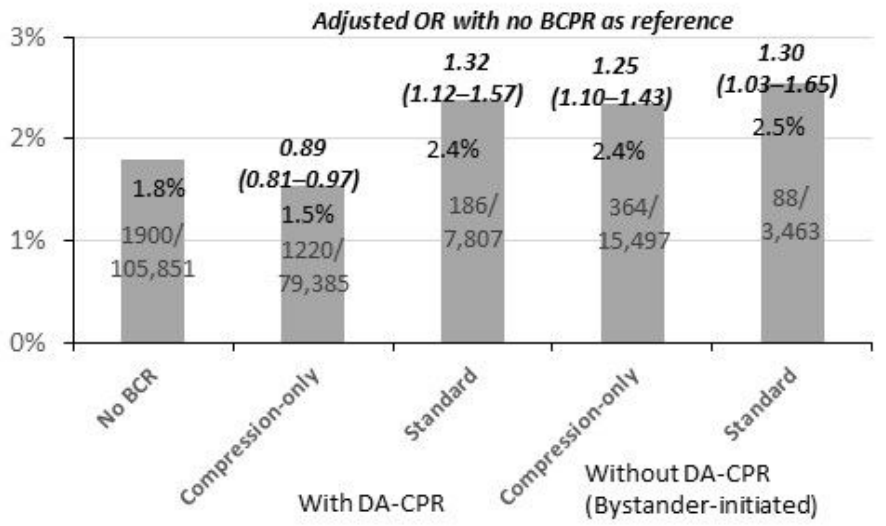

\section{B. Bystander-witnessed OHCAs}

1-M survival

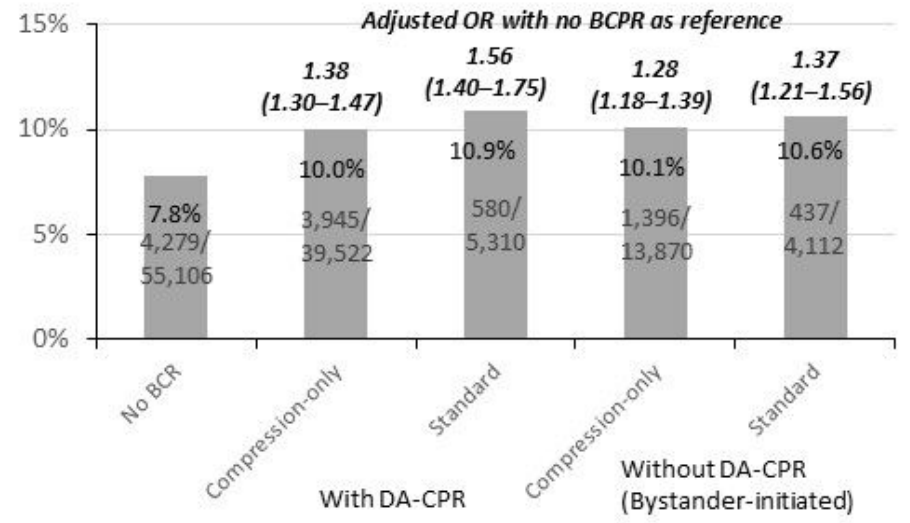

Neurologically favorable outcome

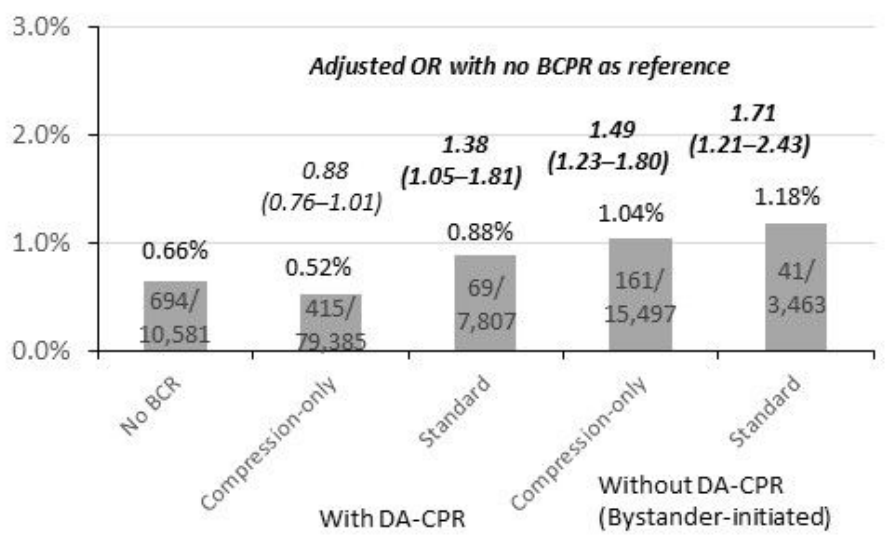

Neurologically favorable outcome

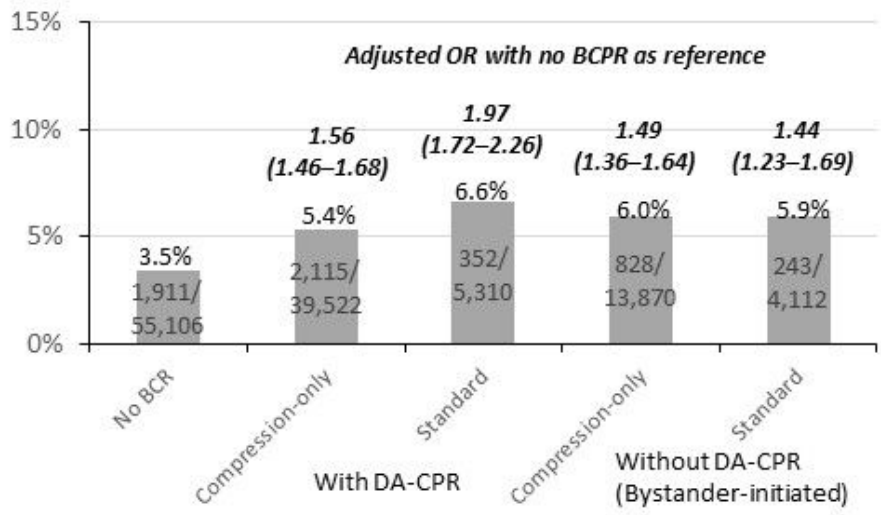

Figure 2

Comparisons of outcomes OHCA: out-of-hospital cardiac arrest, EMS: emergency medical service, BCPR: bystander cardiopulmonary resuscitation, DA-CPR: dispatcher-assisted CPR, OR: odds ratio, Cl: confidence interval, 1-M: 1 month. 


\section{Adjusted OR for Neurologically favorable survival}

Unwitnessed

\section{$B C P R$ with $D A-C P R$}

Combination of breaths (standard $B C P R$ )

Interaction between $B C P R$ with $D A$ -

$C P R$ and combination of ventilations

Age, in $10 y$

Male

Emergency call during nighttime

(10:00 p.m. $-5: 00$ a.m.)

Presumed cardiac etiology

Shockable initial rhythm

PAD

Any prehospital defibrillation

Prehospital epinephrine (no epinephrine $=1$ )

Early single administration

Other administration

Advanced airway management

Physician in ambulance

EMS response time interval, in 1 minute

EMS contact to patient-to-arrival at hospital, in 1 minute

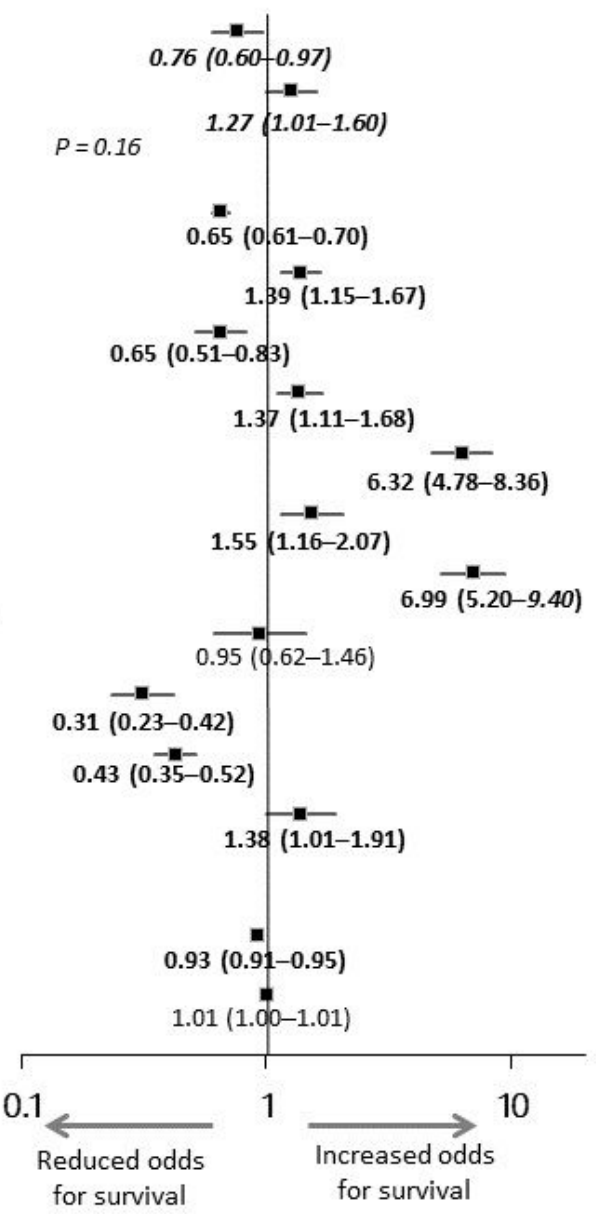

Bystander-witnessed

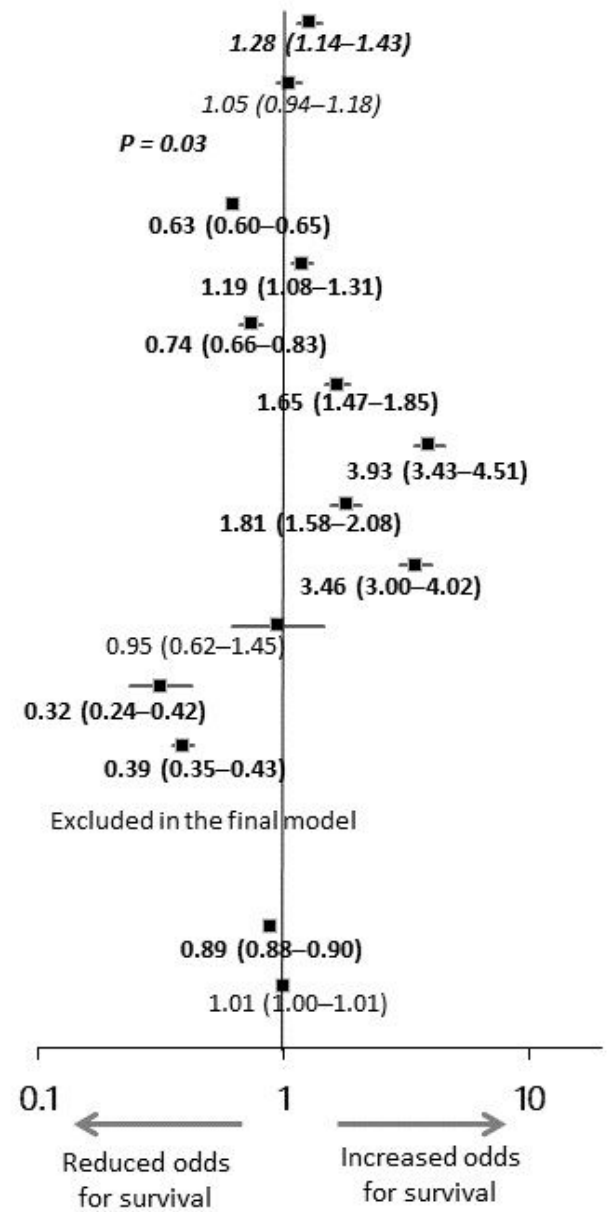

Figure 3

Factors associated with neurologically favourable survival BCPR: bystander cardiopulmonary resuscitation, DA-CPR: dispatcher-assisted CPR, OHCA: out-of-hospital cardiac arrest, PAD: public access defibrillation, ALS: advanced life support, EMS: emergency medical service.

\section{Supplementary Files}

This is a list of supplementary files associated with this preprint. Click to download.

- SupplementalTable1AssociationbetweenclinicalcharacteristicsofbystanderCPRandOHCAinbystander.docx

- SuppleFig.1Final.jpg 